\title{
Fine limits of generalized potential-type integral operators with non-isotropic kernel
}

\author{
Ali Aral
}




\title{
FINE LIMITS OF GENERALIZED POTENTIAL-TYPE INTEGRAL OPERATORS WITH NON-ISOTROPIC KERNEL
}

\author{
ALİ ARAL
}

Received 23 February, 2005

\begin{abstract}
This paper deals with the fine limits of generalized potential-type operators with nonisotropic kernels defined for functions on $\mathbb{R}^{n}$ satisfying appropriate conditions.
\end{abstract}

1991 Mathematics Subject Classification: Primary 31B15; Secondary 31C15, 42B99

Keywords: non-isotropic distance, Riesz potentials

\section{INTRODUCTION}

Let $\lambda_{1}, \lambda_{2}, \ldots, \lambda_{n}$ be positive numbers with $|\lambda|=\lambda_{1}+\lambda_{2}+\cdots+\lambda_{n}$ and $\|x\|_{\lambda}=$ $\left(\left|x_{1}\right|^{\frac{1}{\lambda_{1}}}+\ldots+\left|x_{n}\right|^{\frac{1}{\lambda_{n}}}\right)^{\frac{|\lambda|}{n}}, x \in \mathbb{R}^{n}$. The expression $\|x-y\|_{\lambda}$, where $x, y \in \mathbb{R}^{n}$, is called the $\lambda$-distance or non-isotropic distance between $x$ and $y$. This distance is an important concept in the theory of partial differential equations and imbedding theorems. Some problems with the $\lambda$-distance were examined in $[6,7]$.

It can be seen that $\lambda$-distance becomes the ordinary Euclidean distance $|x-y|$ for $\lambda_{j}=\frac{1}{2}, j=1,2, \ldots, n$. The $\lambda$-distance has the following properties.

Using the inequality $(a+b)^{m} \leq 2^{m}\left(a^{m}+b^{m}\right), m>1$, we obtain

$$
\|x-y\|_{\lambda} \leq M_{\lambda}\left(\|x\|_{\lambda}+\|y\|_{\lambda}\right),
$$

where $M_{\lambda}=2^{\left(1+\frac{1}{\lambda_{\min }}\right) \frac{|\lambda|}{n}}$ and $\lambda_{\min }=\min \left(\lambda_{1}, \lambda_{2}, \ldots \lambda_{n}\right)$.

Several authors have investigated the properties of classical Riesz potentials and their generalizations. For example, taking some appropriate conditions on the kernel depending on Euclidean distance type of $K(|x-y|)$, Gadjiev [3] proved a variant of the Hardy-Littlewood-Sobolev theorem. He also gave the properties of convergence almost everywhere. In [1], a theorem similar to results of [3] was proved for potentialtype integrals with kernel depending on the $\lambda$-distance.

Some results on potential-type integral operators and Riesz potentials given by generalized shift operators can be found in $[2,4,5]$. Various generalizations of the Riesz potentials are given in [10].

Supported by NATO PC-B program of TUBITAK. 
A potential-type integral operator depending on the $\lambda$-distance and defined for non-negative measurable functions $f$ on $\mathbb{R}^{n}$ is given by the equality

$$
(L f)(x)=\int_{\mathbb{R}^{n}} K\left(\|x-y\|_{\lambda}\right) f(y) d y,
$$

where $K$ is the kernel function satisfying the following conditions (see [1]):

$\left(K_{1}\right) K$ is a non-negative continuous and decreasing function on semiaxis $[0, \infty)$ and $\lim _{t \rightarrow 0} K(t)=\infty$;

$\left(K_{2}\right) L(r)=\int_{r}^{a} K\left(2 \beta M_{\lambda} t^{\frac{2|\lambda|}{n}}\right) t^{2|\lambda|-\delta-1} d t<\infty$ for $0<\delta<2|\lambda|, \beta \in(0,1)$ and $0 \leq r<a$.

We know that $(L f)(x) \neq \infty$ if and only if

$$
\int_{\mathbb{R}^{n}} K\left(\beta\left(1+\|y\|_{\lambda}\right)\right) f(y) d y,
$$

where $\beta \in(0,1)$. Hence it is seen that $(L f)(x) \neq \infty$ when $f$ is integrable on $\mathbb{R}^{n}$. Note that (1) is equivalent to

$$
\int_{\mathbb{R}^{n}-B_{\lambda}(x, 1)} K\left(\beta\|x-y\|_{\lambda}\right) f(y) d y
$$

for every $x \in \mathbb{R}^{n}$, and $\beta \in(0,1)$, where $B_{\lambda}(x, 1)$ is $\lambda$-ball centered at $x$ with radius 1. That is $B_{\lambda}(x, 1)=\left\{y \in \mathbb{R}^{n}:\|x-y\|_{\lambda}<1\right\}$.

In what follows, we investigate the fine limits of generalized potential-type integral operators with non-isotropic kernels $L f$ at $x_{0} \in \mathbb{R}^{n}$. Our results are generalizations of the corresponding results for classical Riesz potentials given in [9,11].

To obtain a general result, we assume the condition

$$
\int_{\mathbb{R}^{n}} \phi_{p}(f(y)) w\left(\left\|y-x_{0}\right\|_{\lambda}^{\frac{n}{2|\lambda|}}\right) d y<\infty .
$$

where $x_{0} \in \mathbb{R}^{n}$ and $\phi_{p}(r)$ is positive monotone function on interval $(0, \infty)$ having the following properties:

$\left(\varphi_{1}\right) \phi_{p}(r)$ is of the form $r^{p} \varphi(r)$, where $1 \leq p<\infty$ and $\varphi$ is a positive nondecreasing function on interval $(0, \infty)$.

$\left(\varphi_{2}\right)$ There exists $A_{1}$ such that $\varphi(2 r) \leq A_{1} \varphi(r)$ whenever $r>0$.

Throughout this paper, let $w(r)$ be a positive non-increasing function on $(0, \infty)$ satisfying the condition:

$\left(w_{1}\right)$ There exists $A_{2}>0$ such that $A_{2}^{-1} w(r) \leq w(2 r) \leq A_{2} w(r)$ whenever $r>0$.

In this paper we will use some ideas from $[9,11]$. By the symbol $M$, we denote a positive constant whose value may change depending on the context. 


\section{PRELIMINARY LEMMAS}

First we collect properties which follow from conditions $\left(\varphi_{1}\right)$ and $\left(\varphi_{2}\right)$.

Lemma 2.1. The function $\varphi$ satisfies the doubling condition, that is, there exists $A_{3}>1$ such that

$$
\varphi(r) \leq \varphi(2 r) \leq A_{3} \varphi(r) \quad \text { for } r>0 .
$$

Lemma 2.2. For any $\gamma>0$, there exists $A_{4}(\gamma)>1$ such that

$$
A_{4}^{-1}(\gamma) \varphi(r) \leq \varphi\left(r^{\gamma}\right) \leq A_{4}(\gamma) \varphi(r), \text { whenever } r>0 .
$$

\section{The estimate of $L f$}

We write $(L f)(x)=L_{1}(x)+L_{2}(x)+L_{3}(x)$ for $x \in \mathbb{R}^{n}-\left\{x_{0}\right\}$, where

$$
\begin{aligned}
& L_{1}(x)=\int_{\mathbb{R}^{n}-B_{\lambda}\left(x_{0}, 2 M_{\lambda}\left\|x-x_{0}\right\|_{\lambda}\right)} K\left(\|x-y\|_{\lambda}\right) f(y) d y, \\
& L_{2}(x)=\int_{B_{\lambda}\left(x_{0}, 2 M_{\lambda}\left\|x-x_{0}\right\|_{\lambda}\right)-B_{\lambda}\left(x,\left\|x-x_{0}\right\|_{\lambda} / 2 M_{\lambda}\right)} K\left(\|x-y\|_{\lambda}\right) f(y) d y, \\
& L_{3}(x)=\int_{B_{\lambda}\left(x,\left\|x-x_{0}\right\|_{\lambda} / 2 M_{\lambda}\right)} K\left(\|x-y\|_{\lambda}\right) f(y) d y .
\end{aligned}
$$

Using (1.1), then for any $x, y \in \mathbb{R}^{n}$

$$
\|x-y\|_{\lambda} \geq \frac{1}{M_{\lambda}}\left\|y-x_{0}\right\|_{\lambda}-\left\|x-x_{0}\right\|_{\lambda} .
$$

It is obvious that, if $y \in \mathbb{R}^{n}-B_{\lambda}\left(x_{0}, 2 M_{\lambda}\left\|x-x_{0}\right\|_{\lambda}\right)$, then $\|x-y\|_{\lambda} \geq \frac{1}{2 M_{\lambda}} \| y-$ $x_{0} \|_{\lambda}$. Taking into account $L_{1}(x)$, we have the inequality

$$
L_{1}(x) \leq M \int_{\mathbb{R}^{n}-B_{\lambda}\left(x_{0}, 2 M_{\lambda}\left\|x-x_{0}\right\|_{\lambda}\right)} K\left(\beta\left\|y-x_{0}\right\|_{\lambda}\right) f(y) d y
$$

for any $\beta=\frac{1}{2 M_{\lambda}} \in(0,1)$. For $y \in B_{\lambda}\left(x_{0}, 2 M_{\lambda}\left\|x-x_{0}\right\|_{\lambda}\right)-B_{\lambda}\left(x,\left\|x-x_{0}\right\|_{\lambda} / 2 M_{\lambda}\right)$, since $\|y-x\|_{\lambda} \geq \frac{1}{2 M_{\lambda}}\left\|x-x_{0}\right\|_{\lambda}$, we have similarly

$$
L_{2}(x) \leq K\left(\beta\left\|x-x_{0}\right\|_{\lambda}\right) \int_{B_{\lambda}\left(x_{0}, 2 M_{\lambda}\left\|x-x_{0}\right\|_{\lambda}\right)-B_{\lambda}\left(x,\left\|x-x_{0}\right\|_{\lambda} / 2 M_{\lambda}\right)} f(y) d y
$$

for any $\beta=\frac{1}{2 M_{\lambda}} \in(0,1)$.

Let us begin with the Hölder type inequality. 
Lemma 3.1. Let $p>1, \delta>0$, and $f$ be a non-negative measurable function on $\mathbb{R}^{n}$. If $0 \leq 2 M_{\lambda}\left\|x-x_{0}\right\|_{\lambda}<2 M_{\lambda} a^{\frac{2|\lambda|}{n}}<1$, then

$$
\begin{aligned}
& \int_{\mathbb{R}^{n}-B_{\lambda}\left(x_{0}, 2 M_{\lambda}\left\|x-x_{0}\right\|_{\lambda}\right)} K\left(\beta\left\|y-x_{0}\right\|_{\lambda}\right) f(y) d y \\
& \quad \leq \int_{\mathbb{R}^{n}-B_{\lambda}\left(x_{0}, 2 M_{\lambda} a^{\frac{2|\lambda|}{n}}\right)} K\left(\beta\left\|y-x_{0}\right\|_{\lambda}\right) f(y) d y+M L\left(\left\|x-x_{0}\right\|_{\lambda}^{\frac{n}{2|\lambda|}}\right) \\
& +M R_{1}\left(\left\|x-x_{0}\right\|_{\lambda}^{\frac{n}{2|\lambda|}}\right)\left(\int_{B_{\lambda}\left(x_{0}, 2 M_{\lambda} a^{\frac{2|\lambda|}{n}}\right)} \phi_{p}(f(y)) w\left(\left\|y-x_{0}\right\|_{\lambda}^{\frac{n}{2|\lambda|}}\right) d y\right)^{\frac{1}{p}}
\end{aligned}
$$

where $R_{1}(r)=\left(\int_{r}^{a} K^{p^{\prime}}\left(2 \beta M_{\lambda} t^{\frac{2|\lambda|}{n}}\right)\left[\varphi\left(t^{-1}\right) w(t)\right]^{\frac{p^{\prime}}{p}} t^{2|\lambda|-1} d t\right)^{\frac{1}{p^{\prime}}}$ if $0<2 M_{\lambda} r^{\frac{2|\lambda|}{n}}<$ 1 and $R_{1}(r)=R_{1}\left(\left(2 M_{\lambda}\right)^{-\frac{n}{2|\lambda|}}\right)$ in the other cases.

Proof. Without loss of generality we assume that $f=0$ outside of $B_{\lambda}\left(x_{0}, 2 M_{\lambda} a^{\frac{2|\lambda|}{n}}\right)$. We have

$$
\begin{gathered}
\int_{\mathbb{R}^{n}-B_{\lambda}\left(x_{0}, 2 M_{\lambda}\left\|x-x_{0}\right\|_{\lambda}\right)} K\left(\beta\left\|y-x_{0}\right\|_{\lambda}\right) f(y) d y=\int_{A(y)} K\left(\beta\left\|y-x_{0}\right\|_{\lambda}\right) f(y) d y \\
\leq \int_{\left\{y \in A(y) ; f(y)>\left\|y-x_{0}\right\|_{\lambda}^{\left.-\frac{\delta n}{2|\lambda|}\right\}}\right.} K\left(\beta\left\|y-x_{0}\right\|_{\lambda}\right) f(y) d y \\
+\int_{\left\{y \in A(y) ; f(y) \leq\left\|y-x_{0}\right\|_{\lambda}^{\left.-\frac{\delta n}{2|\lambda|}\right\}}\right.} K\left(\beta\left\|y-x_{0}\right\|_{\lambda}\right) f(y) d y=: L_{11}+L_{12},
\end{gathered}
$$

where $A(y)=B_{\lambda}\left(x_{0}, 2 M_{\lambda} a^{\frac{2|\lambda|}{n}}\right)-B_{\lambda}\left(x_{0}, 2 M_{\lambda}\left\|x-x_{0}\right\|_{\lambda}\right)$. Consider the integral $L_{11}$. From Hölder's inequality, we obtain

$$
\begin{aligned}
L_{11}(x) \leq & \left(\int_{U(y)} f^{p}(y) \varphi(f(y)) w\left(\left\|y-x_{0}\right\|_{\lambda}^{\frac{n}{2 \lambda \mid}}\right) d y\right)^{\frac{1}{p}} \\
& \times\left(\int_{U(y)} K\left(\beta\left\|y-x_{0}\right\|_{\lambda}\right)^{p^{\prime}}\left[\varphi(f(y)) w\left(\left\|y-x_{0}\right\|_{\lambda}^{\frac{n}{2|\lambda|}}\right)\right]^{-\frac{P^{\prime}}{p}} d y\right)^{\frac{1}{p^{\prime}}},
\end{aligned}
$$

where $\frac{1}{p}+\frac{1}{p^{\prime}}=1$ and $U(y)=\left\{y \in A(y) ; f(y)>\left\|y-x_{0}\right\|_{\lambda}^{-\frac{\delta n}{2|\lambda|}}\right\}$. 
Since $\varphi$ is a non-decreasing function, we have $\varphi(f(y)) \geq \varphi\left(\left\|y-x_{0}\right\|_{\lambda}^{-\frac{\delta n}{2|\lambda|}}\right)$ and therefore, Lemma 2.2 implies $\varphi\left(\left\|y-x_{0}\right\|_{\lambda}^{-\frac{\delta n}{2|\lambda|}}\right) \geq M \varphi\left(\left\|y-x_{0}\right\|_{\lambda}^{-\frac{n}{2|\lambda|}}\right)$. Thus,

$$
\begin{aligned}
& L_{11}(x) \leq M\left(\int_{U(y)} \phi_{p}(f(y)) w\left(\left\|y-x_{0}\right\|_{\lambda}^{\frac{n}{2|\lambda|}}\right) d y\right)^{\frac{1}{p}} \\
& \quad \times\left(\int_{U(y)} K\left(\beta\left\|y-x_{0}\right\|_{\lambda}\right)^{p^{\prime}}\left[\varphi\left(\left\|y-x_{0}\right\|_{\lambda}^{-\frac{n}{2|\lambda|}}\right) w\left(\left\|y-x_{0}\right\|_{\lambda}^{\frac{n}{2|\lambda|}}\right)\right]^{-\frac{P^{\prime}}{p}} d y\right)^{\frac{1}{p^{\prime}}} .
\end{aligned}
$$

The right hand side integral with respect to $y$ may be easily calculated. Namely, passing to generalized spherical coordinates by transformation

$$
\begin{aligned}
y_{1} & =x_{01}+\left(t \cos \theta_{1}\right)^{2 \lambda_{1}}, \\
y_{2} & =x_{02}+\left(t \sin \theta_{1} \cos \theta_{2}\right)^{2 \lambda_{2}}, \\
& \vdots \\
y_{n} & =x_{0 n}+\left(t \sin \theta_{1} \sin \theta_{2} \ldots \sin \theta_{n-1}\right)^{2 \lambda_{n},}
\end{aligned}
$$

where $\theta_{j}, j=1,2, \ldots, n$, are the coordinates of the point $\theta$ on unit sphere. We can see that the Jacobian of this transformation $t^{2|\lambda|-1} \Omega_{\lambda}(\theta)$, where $\Omega_{\lambda}(\theta)$ depends on angles $\theta_{1}, \theta_{2}, \ldots, \theta_{n-1}$ only $0 \leq \theta_{1}, \ldots, \theta_{n-2} \leq \pi, 0 \leq \theta_{n-1} \leq 2 \pi$ and

$$
\Omega_{\lambda}(\theta)=2^{n} \prod_{j=1}^{n-1}\left(\cos \theta_{j}\right)^{2 \lambda_{j}-1}\left(\sin \theta_{j}\right)^{2|\lambda|-\sum_{k=1}^{j} \lambda_{k}-1} .
$$

Here the integral $\int_{S^{n-1}} \Omega_{\lambda}(\theta) d \theta$ is finite, where $S^{n-1}$ is the unit ball in $\mathbb{R}^{n}$. Consequently, from (3.3) we have

$$
\begin{array}{r}
L_{11}(x) \leq M\left(\int_{\left(2 M_{\lambda}\right)^{\frac{n}{2|\lambda|}}\left\|x-x_{0}\right\|_{\lambda}^{\frac{n}{2 \lambda \mid}}}^{\left(2 M_{\lambda}\right)^{\frac{n}{2|\lambda|}} a} K^{p^{\prime}}\left(\beta t^{\frac{2|\lambda|}{n}}\right)\left[\varphi\left(t^{-1}\right) w(t)\right]^{-\frac{p^{\prime}}{p}} t^{2|\lambda|-1} d t\right)^{\frac{1}{p^{\prime}}} \\
\times\left(\int_{U(y)} \phi_{p}(f(y)) w\left(\left\|y-x_{0}\right\|_{\lambda}^{\frac{n}{2 \lambda \mid}}\right) d y\right)^{\frac{1}{p}} \cdot
\end{array}
$$


Let us now consider the integral $L_{12}$. By passing to generalized spherical coordinates, we get

$$
\begin{aligned}
L_{12}(x) & \leq \int_{A(y)} K\left(\left\|y-x_{0}\right\|_{\lambda}\right)\left\|y-x_{0}\right\|_{\lambda}^{-\frac{n}{2|\lambda|} \delta} d y \\
& =M L\left(\left\|x-x_{0}\right\|_{\lambda}^{\frac{n}{2|\lambda|}}\right),
\end{aligned}
$$

where $L(r)$ is defined in the condition $\left(K_{2}\right)$. Relations (3.4) and (3.5) give the desired conclusion.

Lemma 3.2. Let $f$ be a non-negative measurable function on $\mathbb{R}^{n}$. If $0<2 M_{\lambda} \| x-$ $x_{0} \|_{\lambda}<1$ and $0<\delta<2|\lambda|$, then there exists a positive $M$ such that

$$
\begin{gathered}
L_{2}(x) \leq M R_{2}\left(\left\|x-x_{0}\right\|_{\lambda}^{\frac{n}{2|\lambda|}}\right)\left(\int_{B_{\lambda}\left(x_{0}, 2 M_{\lambda}\left\|x-x_{0}\right\|_{\lambda}\right)} \phi_{p}(f(y)) w\left(\left\|y-x_{0}\right\|_{\lambda}^{\frac{n}{2|\lambda|}}\right) d y\right)^{\frac{1}{p}} \\
+M\left\|x-x_{0}\right\|_{\lambda}^{2|\lambda|-\delta}
\end{gathered}
$$

where

$$
R_{2}(r)=K\left(\beta t^{\frac{2|\lambda|}{n}}\right) r^{\frac{2|\lambda|}{n} \frac{2|\lambda|}{p^{\prime}}}\left[\varphi\left(r^{-\frac{2|\lambda|}{n}}\right) w(r)\right]^{-\frac{1}{p}} .
$$

Proof. It follows from (3.2) that

$$
\begin{aligned}
L_{2}(x) \leq & K\left(\beta\left\|x-x_{0}\right\|_{\lambda}\right) \int_{B(x)} f(y) d y \\
\leq & K\left(\beta\left\|x-x_{0}\right\|_{\lambda}\right)\left\{\int_{\left\{y \in B(x) ; f(y)>\left\|x-x_{0}\right\|_{\lambda}^{-\delta\}}\right.} f(y) d y\right. \\
& \left.\quad+\int_{\left\{y \in B(x) ; f(y) \leq\left\|x-x_{0}\right\|_{\lambda}^{-\delta}\right\}} f(y) d y\right\}=: L_{21}(x)+L_{22}(x),
\end{aligned}
$$

where $B(x)=B_{\lambda}\left(x_{0}, 2 M_{\lambda}\left\|x-x_{0}\right\|_{\lambda}\right)-B_{\lambda}\left(x,\left\|x-x_{0}\right\|_{\lambda} / 2 M_{\lambda}\right)$.

Let us first consider $L_{21}$. Since $\varphi$ is a non-decreasing function, by Lemma 3.1, we get

$$
L_{21}(x) \leq M\left[\varphi\left(\left\|x-x_{0}\right\|_{\lambda}^{-1}\right)\right]^{-\frac{1}{p}} K\left(\beta\left\|x-x_{0}\right\|_{\lambda}\right) \int_{B(x)} f(y)[\varphi(f(y))]^{\frac{1}{p}} d y .
$$

From Hölder's inequality, we obtain

$$
\begin{aligned}
L_{21}(x) \leq M[\varphi( & \left.\left.\left\|x-x_{0}\right\|_{\lambda}^{-1}\right)\right]^{-\frac{1}{p}} K\left(\beta\left\|x-x_{0}\right\|_{\lambda}\right) \\
& \times\left(\int_{B_{\lambda}\left(x_{0}, 2 M_{\lambda}\left\|x-x_{0}\right\|_{\lambda}\right)} d y\right)^{\frac{1}{p^{\prime}}}\left(\int_{B(x)} f(y)^{p} \varphi(f(y)) d y\right)^{\frac{1}{p}},
\end{aligned}
$$

where $\frac{1}{p}+\frac{1}{p^{\prime}}=1$. 
Therefore, because $w$ is a non-increasing function, it follows that

$$
\begin{aligned}
L_{21}(x) \leq M & {\left[\varphi\left(\left\|x-x_{0}\right\|_{\lambda}^{-1}\right)\right]^{-\frac{1}{p}} K\left(\beta\left\|x-x_{0}\right\|_{\lambda}\right)\left\|x-x_{0}\right\|_{\lambda}^{\frac{2|\lambda|}{P^{\prime}}} } \\
& \times\left(\int_{B(x)} \phi_{p}(f(y)) d y\right)^{\frac{1}{p}} \\
\leq & M\left(\varphi\left(\left\|x-x_{0}\right\|_{\lambda}^{-1}\right) w\left(\left\|x-x_{0}\right\|_{\lambda}^{\frac{n}{2|\lambda|}}\right)\right)^{-\frac{1}{p}} K\left(\beta\left\|x-x_{0}\right\|_{\lambda}\right)\left\|x-x_{0}\right\|_{\lambda}^{\frac{2|\lambda|}{P^{\prime}}} \\
& \times\left(\int_{B_{\lambda}\left(x_{0}, 2 M_{\lambda}\left\|x-x_{0}\right\|_{\lambda}\right)} \phi_{p}(f(y)) w\left(\left\|y-x_{0}\right\|_{\lambda}^{\frac{n}{2|\lambda|}}\right) d y\right)^{\frac{1}{p}}
\end{aligned}
$$

On the other hand, we have

$$
\begin{aligned}
L_{22} & \leq K\left(\beta\left\|x-x_{0}\right\|_{\lambda}\right) \int_{B_{\lambda}\left(x_{0}, 2 M_{\lambda}\left\|x-x_{0}\right\|_{\lambda}\right)}\left\|x-x_{0}\right\|_{\lambda}^{-\delta} d y \\
& \leq M K\left(\beta\left\|x-x_{0}\right\|_{\lambda}\right)\left\|x-x_{0}\right\|_{\lambda}^{2|\lambda|-\delta} .
\end{aligned}
$$

We have the desired conclusion from (3.7) and (3.8).

Lemma 3.3. Let $f$ be a non-negative measurable function on $\mathbb{R}^{n}$. If $\delta>0$, then there exists a positive $M$ such that

$$
\begin{aligned}
L_{3}(x) \leq M R_{3}\left(\left\|x-x_{0}\right\|_{\lambda}^{\frac{n}{2|\lambda|}}\right) & \left(\int_{B_{\lambda}\left(x,\left\|x-x_{0}\right\|_{\lambda} / 2 M_{\lambda}\right)} \phi_{p}(f(y)) w\left(\left\|y-x_{0}\right\|_{\lambda}^{\frac{n}{2|\lambda|}}\right) d y\right)^{\frac{1}{p}} \\
& +M \int_{0}^{\left(\left\|x-x_{0}\right\|_{\lambda} / 2 M_{\lambda}\right)^{\frac{n}{2|\lambda|}}} K^{p^{\prime}}\left(2 \beta M_{\lambda} t^{\frac{2|\lambda|}{n}}\right) t^{2|\lambda|-\delta-1} d t
\end{aligned}
$$

where $R_{3}(r)=\varphi^{*}(r) \omega(r)^{-\frac{1}{p}}$ and $\varphi^{*}(r)=\left(\int_{0}^{r} K^{p^{\prime}}\left(t^{\frac{2|\lambda|}{n}}\right)\left[\varphi\left(t^{-1}\right)\right]^{-\frac{p^{\prime}}{p}} t^{2|\lambda|-1} d t\right)^{\frac{1}{p^{\prime}}}$

Proof. By change of variable, we have

$$
L_{3}(x)=\int_{B_{\lambda}\left(0,\left\|x-x_{0}\right\|_{\lambda} / 2 M_{\lambda}\right)} K\left(\|y\|_{\lambda}\right) f(x+y) d y .
$$


In a way similar to the proof of Lemmas 3.1 and 3.2, we obtain

$$
\begin{aligned}
L_{3}(x) \leq M & \left(\int_{0}^{\left(\left\|x-x_{0}\right\|_{\lambda} / 2 M_{\lambda}\right)^{\frac{n}{2|\lambda|}}} K^{p^{\prime}}\left(t^{\frac{2|\lambda|}{n}}\right)\left[\varphi\left(t^{-1}\right)\right]^{-\frac{p^{\prime}}{p}} t^{2|\lambda|-1} d t\right)^{\frac{1}{p^{\prime}}} \\
& \times\left(\int_{B_{\lambda}\left(0,\left\|x-x_{0}\right\|_{\lambda} / 2 M_{\lambda}\right)} \phi_{p}(f(x+y)) d y\right)^{\frac{1}{p}} \\
& +M \int_{0}^{\left(\left\|x-x_{0}\right\|_{\lambda} / 2 M_{\lambda}\right)^{\frac{n}{2|\lambda|}}} K\left(\beta 2 M_{\lambda} t^{\frac{2|\lambda|}{n}}\right) t^{2|\lambda|-\delta-1} d t \\
\leq & M \varphi^{*}\left(\left\|x-x_{0}\right\|_{\lambda}^{\frac{n}{2 \lambda \mid \lambda}}\right) w\left(\left\|x-x_{0}\right\|_{\lambda}^{\frac{n}{2|\lambda|}}\right)^{-\frac{1}{p}} \\
& \times\left(\int_{B_{\lambda}\left(x,\left\|x-x_{0}\right\|_{\lambda} / 2 M_{\lambda}\right)} \phi_{p}(f(y)) w\left(\left\|y-x_{0}\right\|_{\lambda}^{\frac{n}{2|\lambda|}}\right) d y\right)^{\frac{1}{p}} \\
& +\int_{0}^{\left(\left\|x-x_{0}\right\|_{\lambda} / 2 M_{\lambda}\right)^{\frac{n}{2|\lambda|}}} K\left(\beta 2 M_{\lambda} t^{\frac{2|\lambda|}{n}}\right) t^{2|\lambda|-\delta-1} d t
\end{aligned}
$$

as required.

\section{Fine Limit of $R_{\alpha} f$}

We consider the function

$$
\begin{aligned}
R(r) & =R_{1}(r)+R_{2}(r)+R_{3}(r) \\
& =R_{1}(r)+K\left(\beta t^{\frac{2|\lambda|}{n}}\right) r^{\frac{2|\lambda|}{n} \frac{2|\lambda|}{p^{\prime}}}\left(w(r) \varphi\left(r^{-\frac{2|\lambda|}{n}}\right)\right)^{-\frac{1}{p}}+\varphi^{*}(r) w(r)^{-\frac{1}{p}} .
\end{aligned}
$$

Theorem 4.1. Let $p>1$ and $f$ be a non-negative measurable function on $\mathbb{R}^{n}$ satisfying conditions (1.2) and (1.3). If $\varphi^{*}(1)<\infty$ and $\lim _{r \rightarrow 0} R(r)=\infty$, then

$$
\lim _{x \rightarrow x_{0}}\left[R\left(\left\|x-x_{0}\right\|_{\lambda}\right)\right]^{-1}(L f)(x)=0 .
$$

If $R(r)$ is bounded, then $(L f)\left(x_{0}\right)$ is finite and $(L f)(x)$ tends to $(L f)\left(x_{0}\right)$ as $x \rightarrow$ $x_{0}$.

Proof. By condition (1.2), the integral

$$
\int_{\mathbb{R}^{n}-B_{\lambda}\left(x_{0}, 2 M_{\lambda} a^{\frac{2|\lambda|}{n}}\right)} K\left(\beta\left\|y-x_{0}\right\|_{\lambda}\right) f(y) d y
$$


is finite. It follows from (3.1), the condition $K_{2}$ and Lemma 3.1 that

$$
\begin{aligned}
& \limsup _{x \rightarrow x_{0}}\left(R\left(\left\|x-x_{0}\right\|_{\lambda}^{\frac{n}{2|\lambda|}}\right)\right)^{-1} L_{1}(x) \\
& \quad \leq M\left(\int_{B_{\lambda}\left(x_{0}, 2 M_{\lambda} a^{\frac{2|\lambda|}{n}}\right)} \phi_{p}(f(y)) w\left(\left\|y-x_{0}\right\|_{\lambda}^{\frac{n}{2|\lambda|}}\right) d y\right)^{\frac{1}{p}} .
\end{aligned}
$$

Since $a$ is arbitrary, we see that the integral in the left-hand side of the last estimate is equal to zero.

In view of Lemmas 3.2 and 3.3 and condition (1.3), we have

$$
\lim _{x \rightarrow x_{0}}\left[R\left(\left\|x-x_{0}\right\|_{\lambda}\right)\right]^{-1}\left(L_{2}(x)+L_{3}(x)\right)=0 .
$$

If we combine these results, we have

$$
\lim _{x \rightarrow x_{0}}\left[R\left(\left\|x-x_{0}\right\|_{\lambda}\right)\right]^{-1}(L f)(x)=0 .
$$

If $R(r)$ is bounded, then Lemmas 3.2 and 3.3 imply that $L_{2}(x)+L_{3}(x)$ tends to zero at $x \rightarrow x_{0}$. Furthermore, in view of Lemma 3.1, we have $\limsup _{x \rightarrow x_{0}} L_{1}(x)<$ $\infty$. Thus it follows that $(L f)\left(x_{0}\right)$ is finite. Hence

$$
L_{1}(x)+L_{2}(x)=\int_{\mathbb{R}^{n}-B_{\lambda}\left(x,\left\|x-x_{0}\right\|_{\lambda} / 2 M_{\lambda}\right)} K\left(\|x-y\|_{\lambda}\right) f(y) d y .
$$

Since $\left\|y-x_{0}\right\|_{\lambda} \leq 2 M_{\lambda}^{2}\|y-x\|_{\lambda}$ for $y \in \mathbb{R}^{n}-B_{\lambda}\left(x,\left\|x-x_{0}\right\|_{\lambda} / 2 M_{\lambda}\right)$, we have by Lebesgue's dominated convergence theorem

$$
\lim _{x \rightarrow x_{0}}\left(L_{1}(x)+L_{2}(x)\right)=(L f)\left(x_{0}\right) .
$$

However, we also know that $\lim _{x \rightarrow x_{0}} L_{3}(x)=0$. The proof of Theorem 4.1 is thus complete.

Corollary 4.1 ([8,9]). Let $p=\frac{n}{\alpha}$ and $\varphi^{*}(1)<\infty$. If $f$ is a non-negative measurable function on $\mathbb{R}^{n}$ satisfying (1.2) and the condition

$$
\int_{\mathbb{R}^{n}} \phi_{p}(f(y)) d y<\infty
$$

then $L_{\alpha} f$ is continuous on $\mathbb{R}^{n}$ with $K(t)=t^{\alpha-n}, 0<\alpha<n$, and $\lambda_{k}=\frac{1}{2}, k=$ $1,2, \ldots, n$.

Corollary 4.2 ([1]). Let $f$ be a non-negative measurable function satisfying conditions (1.2) and the condition

$$
\int_{\mathbb{R}^{n}} \phi_{p}(f(y)) d y<\infty
$$

then $L_{\alpha} f$ is continuous on $\mathbb{R}^{n}$. 
Proposition 4.1. Let ap $=n, \varphi^{*}(1)<\infty, x_{0}=0, K(t)=t^{\alpha-n}$, and

$$
\lim _{r \rightarrow 0} r^{\frac{2|\lambda|}{p^{\prime}}}(w(r))^{-\frac{1}{p}}\left(\varphi\left(r^{-1}\right)\right)^{-\frac{1}{p}}=0 .
$$

Then for any positive non-decreasing function $a(r)$ on $(0, \infty)$ such that

$$
\lim _{r \rightarrow 0} a(r)=\infty
$$

there exists a non-negative measurable function $f$ satisfying (1.2) and (1.3) such that

$$
\limsup _{x \rightarrow x_{0}} a\left(\|x\|_{\lambda}^{\frac{n}{2|\lambda|}}\right)\left(w\left(\|x\|_{\lambda}^{\frac{n}{2|\lambda|}}\right) \varphi\left(\|x\|_{\lambda}^{-\frac{n}{2|\lambda|}}\right)\right)^{-\frac{1}{p}} R_{\alpha} f(x)=\infty
$$

where $\frac{1}{p}+\frac{1}{p^{\prime}}=1$.

Proof. Let $\left(j_{i}\right)$ be a sequence of positive integers such that $j_{i}+2<J_{i+1}$ and $\sum_{i} a_{i}^{-\frac{1}{p}}<\infty$, where $a_{i}\left(r_{j}\right)=a_{i}$ and $r_{j}=2^{-j_{i}}$. We set

$$
f(y)=a_{i}^{-\frac{1}{p}}\left(\varphi\left(r_{j}^{-1}\right)\right)^{\frac{1}{p^{\prime}}}\left(w\left(r_{j}\right)\right)^{-\frac{1}{p}}\left\|x_{i}-y\right\|_{\lambda}^{-\alpha}\left[\varphi\left(\left\|x_{i}-y\right\|_{\lambda}^{-1}\right)\right]^{-1}
$$

if $y \in \bigcup_{i=1}^{\infty} B_{\lambda}\left(x_{i},\left(2 r_{j}\right)^{\frac{2|\lambda|}{n}}\right)-B_{\lambda}\left(x_{i},\left(r_{j}\right)^{\frac{2|\lambda|}{n}}\right)$, otherwise $f(y)=0$, where $x_{i}=$ $\left(r_{j}, 0, \ldots, 0\right) \in \mathbb{R}^{n}$.

Let us now show that $f$ meets all the conditions in the proposition. If we use Lemmas 2.1 and 2.2, then we have

$$
\begin{aligned}
\int f(y) d y= & \sum_{i} a_{i}^{-\frac{1}{p}}\left(\varphi\left(r_{j}^{-1}\right)\right)^{\frac{1}{p^{\prime}}}\left(w\left(r_{j}\right)\right)^{-\frac{1}{p}} \\
& \times \int_{B_{\lambda}\left(x_{i},\left(2 r_{j}\right)^{\frac{2|\lambda|}{n}}\right)-B_{\lambda}\left(x_{i},\left(r_{j}\right)^{\frac{2|\lambda|}{n}}\right)}\left\|x_{i}-y\right\|_{\lambda}^{-\alpha}\left[\varphi\left(\left\|x_{i}-y\right\|_{\lambda}^{-1}\right)\right]^{-1} d y \\
\leq & M \sum_{i} a_{i}^{-\frac{1}{p}}\left(\varphi\left(r_{j}^{-1}\right)\right)^{\frac{1}{p^{\prime}}}\left(w\left(r_{j}\right)\right)^{-\frac{1}{p}} \int_{r_{j}}^{2 r_{j}} t^{-\frac{2|\lambda|}{n} \alpha}\left(\varphi\left(t^{-\frac{2|\lambda|}{n}}\right)\right)^{-1} t^{2|\lambda|-1} d t \\
\leq & M \sum_{i} a_{i}^{-\frac{1}{p}}\left\{r_{j}^{\frac{2|\lambda|}{p^{\prime}}}\left(\varphi\left(r_{j}^{-1}\right)\right)^{\frac{1}{p}}\left(w\left(r_{j}\right)\right)^{-\frac{1}{p}}\right\} \\
\leq & M \sum_{i} a_{i}^{-\frac{1}{p}}<\infty .
\end{aligned}
$$


Consequently $f$ satisfies (1.2). On the other hand, since the values $\left(a_{i}^{-\frac{1}{p}}\right)$ and $\left(r_{j}^{\frac{2|\lambda|}{p^{\prime}}}\left(\varphi\left(r_{j}^{-1}\right)\right)^{-\frac{1}{p}}\left(w\left(r_{j}\right)\right)^{-\frac{1}{p}}\right)$ are bounded, we have

$$
\begin{aligned}
f(y) & \leq M\left(\varphi\left(r_{j}^{-1}\right)\right)^{\frac{1}{p^{\prime}}}\left(w\left(r_{j}\right)\right)^{-\frac{1}{p}}\left\|x_{i}-y\right\|_{\lambda}^{-\alpha}\left[\varphi\left(\left\|x_{i}-y\right\|_{\lambda}\right)\right]^{-1} \\
& \leq M\left(\varphi\left(r_{j}^{-1}\right)\right)^{-\frac{1}{p}}\left\{r_{j}^{\frac{2|\lambda|}{p^{\prime}}} \varphi\left(r_{j}^{-1}\right)^{-\frac{1}{p}}\left\|x_{i}-y\right\|_{\lambda}^{-\alpha}\right\}^{-1} \\
& \leq M\left\|x_{i}-y\right\|_{\lambda}^{-\alpha-\frac{n}{p^{\prime}}} .
\end{aligned}
$$

Thus, the inequality $\varphi(f(y)) \leq \varphi\left(\left\|x_{i}-y\right\|_{\lambda}^{-1}\right)$ holds.

Now we show that $f$ satisfies (1.3). Using condition $\left(w_{1}\right)$, we get

$$
\begin{aligned}
& \int_{\mathbb{R}^{n}} \phi_{p}(f(y)) w\left(\|y\|_{\lambda}^{\frac{n}{2|\lambda|}}\right) d y \\
& \leq \sum_{i} a_{i}^{-1}\left(\varphi\left(r_{j}^{-1}\right)\right)^{\frac{p}{p^{\prime}}} \int_{B_{\lambda}\left(x_{i},\left(2 r_{j}\right)^{\frac{2|\lambda|}{n}}\right)-B_{\lambda}\left(x_{i},\left(r_{j}\right)^{\frac{2|\lambda|}{n}}\right)}\left\|x_{i}-y\right\|_{\lambda}^{-\alpha p} \\
& \times\left[\varphi\left(\left\|x_{i}-y\right\|_{\lambda}^{-1}\right)\right]^{-\frac{p}{p^{\prime}}} d y \\
& \leq M \sum_{i} a_{i}^{-1}\left(\varphi\left(r_{j}^{-1}\right)\right)^{\frac{p}{p^{\prime}}} \int_{r_{j}}^{2 r_{j}} t^{-\frac{2|\lambda|}{n} \alpha p}\left(\varphi\left(t^{-\frac{2|\lambda|}{n}}\right)\right)^{-\frac{p}{p^{\prime}}} t^{2|\lambda|-1} d t \\
& \leq M \sum_{i} a_{i}^{-1}\left(\varphi\left(r_{j}^{-1}\right)\right)^{\frac{p}{p^{\prime}}} \int_{r_{j}}^{2 r_{j}}\left(\varphi\left(t^{-1}\right)\right)^{-\frac{p}{p^{\prime}}} t^{-1} d t \\
& \leq M \sum_{i} a_{i}^{-1}<\infty .
\end{aligned}
$$

Finally,

$$
\begin{aligned}
R_{\alpha} f\left(x_{i}\right) \geq & a_{i}^{-\frac{1}{p}}\left(\varphi\left(r_{j}^{-1}\right)\right)^{\frac{1}{p^{\prime}}}\left(w\left(r_{j}\right)\right)^{-\frac{1}{p}} \\
& \times \int_{B_{\lambda}\left(x_{i},\left(2 r_{j}\right)^{\frac{2|\lambda|}{n}}\right)-B_{\lambda}\left(x_{i},\left(r_{j}\right)^{\frac{2|\lambda|}{n}}\right)}\left\|x_{i}-y\right\|_{\lambda}^{-n}\left[\varphi\left(\left\|x_{i}-y\right\|_{\lambda}^{-1}\right)\right]^{-1} d y \\
\geq & M a_{i}^{-\frac{1}{p}}\left(\varphi\left(r_{j}^{-1}\right)\right)^{\frac{1}{p^{\prime}}}\left(w\left(r_{j}\right)\right)^{-\frac{1}{p}} \int_{r_{j}}^{2 r_{j}}\left(\varphi\left(t^{-1}\right)\right)^{-1} t^{-1} d t \\
\geq & M a_{i}^{-\frac{1}{p}}\left(\varphi\left(r_{j}^{-1}\right)\right)^{-\frac{1}{p}}\left(w\left(r_{j}\right)\right)^{-\frac{1}{p}} .
\end{aligned}
$$

Thus we have

$$
a_{i}\left(\varphi\left(r_{j}^{-1}\right)\right)^{\frac{1}{p}}\left(w\left(r_{j}\right)\right)^{\frac{1}{p}} R_{\alpha} f\left(x^{(i)}\right) \geq M a_{i}^{\frac{1}{p}} .
$$

This proves the proposition for $j \rightarrow \infty$. 


\section{REFERENCES}

[1] A. Aral, "On generalized potentials-type integral operators with non-isotropic kernels," Int. Math. J., vol. 3, no. 12, pp. 1367-1378, 2003.

[2] A. D. Gadjiev and I. A. Aliyev, "Riesz and Bessel potentials generalized by generalized shift operators and its inversion," in Theory of Functions and Approximation. Proc. IV Math. Conference, Saratov, 1988, Saratov, 1990, pp. 43-53.

[3] A. D. Gadjiev, "On generalized potential-type integral operators," Funct. Approx. Comment. Math., vol. 25, pp. 37-44, 1997, dedicated to Roman Taberski on the occasion of his 70th birthday.

[4] V. S. Guliev and Z. V. Safarov, "On generalized fractional integrals, associated with the Bessel differential expansions," Trans. Acad. Sci. Azerb. Ser. Phys.-Tech. Math. Sci., vol. 22, no. 4, Math. Mech., pp. 75-90, 2002, VIII Republic Conference on Mathematics and Mechanics (Baku, 2002).

[5] V. S. Guliyev and R. C. Mustafayev, "On generalized fractional integrals," Trans. Acad. Sci. Azerb. Ser. Phys.-Tech. Math. Sci., vol. 21, no. 4, Math. Mech., pp. 63-71, 237, 2001.

[6] N. S. Landkof, Foundations of modern potential theory. New York: Springer-Verlag, 1972, translated from the Russian by A. P. Doohovskoy, Die Grundlehren der mathematischen Wissenschaften, Band 180.

[7] P. I. Lizorkin, "Multipliers of Fourier integrals and estimates of convolutions in spaces with mixed norm. Applications," Izv. Akad. Nauk SSSR Ser. Mat., vol. 34, pp. 218-247, 1970.

[8] Y. Mizuta, "Continuity properties of Riesz potentials and boundary limits of Beppo Levi functions," Math. Scand., vol. 63, no. 2, pp. 238-260, 1988.

[9] Y. Mizuta, "Continuity properties of potentials and Beppo-Levi-Deny functions," Hiroshima Math. J., vol. 23, no. 1, pp. 79-153, 1993.

[10] E. Nakai and H. Sumitomo, "On generalized Riesz potentials and space of some smooth functions," preprint.

[11] T. Shimomura and Y. Mizuta, "Taylor expansion of Riesz potentials," Hiroshima Math. J., vol. 25, no. 3, pp. 595-621, 1995.

\section{Author's address}

\section{Ali Aral}

Kırıkkale University, Faculty of Science and Arts, Department of Mathematics, Yahşihan, Kirikkale, Turkey

E-mail address: aralescience.ankara.edu.tr 